\title{
Elektronisches Patientendossier durchläuft eine kritische Phase
}

\section{Yvonne Gilli}

Dr. med., Mitglied des FMH-Zentralvorstandes, Departementsverantwortliche Digitalisierung / eHealth



Zurzeit arbeiten das Bundesamt für Gesundheit (BAG) und eHealth Suisse als Koordinationsorgan zwischen Bund und Kantonen intensiv an den Ausführungsbestimmungen zum Gesetz über das elektronische Patientendossier (EPD). Der Zeitplan ist ambitiös. Spätestens im ersten Quartal 2017 sollten die Ausführungsverordnungen abgeschlossen sein. Das Gesetz über das elektronische Patientendossier kann zu diesem Zeitpunkt in Kraft gesetzt werden.

Die Frist für die Stellungnahme zum ersten Entwurf des Ausführungsrechtes ist abgelaufen. Die FMH hat ausführlich und dezidiert kritisch Stellung genommen. Sämtliche Stellungnahmen sind auf der BAGHomepage aufgeschaltet und einsehbar. Was wir befürchtet haben, ist eingetreten: Die Regelungsdichte und die Anforderungen sind zu hoch. Die Ausführungsbestimmungen sind wegen der umfangreichen technischen und juristischen Detailregelungen schwer lesbar und nicht zweckmässig. Für die Ärztinnen und Ärzte besteht das elektronische Patientendossier die Feuertaufe nur, wenn die notwendigen Prozesse im Arbeitsalltag geklärt und einbezogen werden. Das ist bis jetzt nicht geschehen.

\section{Regelungsdichte und Anforderungen des elektronischen Patientendossiers sind zu hoch.}

Die Verantwortlichen im BAG haben die breite Kritik wahrgenommen und sind bereit, wesentliche Punkte der Verordnung zu überarbeiten. Um den Praxisbezug und den Nutzen für Patienten und Gesundheitsberufe $\mathrm{zu}$ sichern, braucht es jetzt die vereinte Kraft der betroffenen Berufsverbände. Die Interprofessionelle Arbeitsgruppe elektronisches Patientendossier (IPAG EPD) spielt eine Schlüsselrolle. In der IPAG haben sich 2014 acht nationale Berufsverbände zusammengeschlossen - ChiroSuisse, Ergotherapeutinnen und -therapeuten, Ernährungsberaterinnen und -berater, FMH, Hebammen, pharmaSuisse, physiosuisse und SBK.

Die IPAG steht unter einem enormen Druck. Diese breite interprofessionelle Zusammenarbeit gibt es erst zwei Jahre. Eine gemeinsame Kommunikationskultur und die Entwicklung solidarischer Verantwortlichkeit brauchen Zeit und die Freiheit, sich zuerst kleinen gemeinsamen Handlungsfeldern zu widmen. Beide Voraussetzungen sind bei der Ausgestaltung des elektronischen Patientendossiers nicht gegeben.

Bereits diesen Sommer musste die IPAG gegenüber eHealth Suisse und dem BAG erste Resultate liefern. Es war ein Kraftakt mit Bewährungsprobe! Alle beteiligten Verbände mussten Ressourcen mobilisieren, welche die übliche Kapazität ihrer Dienstleistungen überschritten. Die IPAG schaffte es, einen Konsensbericht zu verabschieden, welcher interprofessionelle Behandlungsprozesse am Beispiel der eMedikation aufzeigt und mit einem Patientenbeispiel veranschaulicht.

Notwendig ist die Konzentration auf zentrale Eckpunkte, welche gemeinsam mit den Leistungserbringern festzulegen sind.

Die eMedikation ist ein elektronisches Informationssystem über Medikationsprozesse. Teile der eMedikation sind international vergleichsweise gut erprobt und standardisiert. Die eMedikation wird deshalb einer der ersten Bestandteile des elektronischen Patientendossiers darstellen. Zentrale Elemente der eMedikation sind aus Sicht der IPAG die Übersicht über die aktuelle Medikation, das elektronische Rezept, Informationen zu Abgabe und Anwendung sowie die Möglichkeit eines (Freitext)-Kommentars.

Was es jetzt von Seiten des BAG braucht, ist eine Verlangsamung im Umsetzungsprozess und die Konzentration auf wesentliche Eckpunkte, welche nur zusammen mit den betroffenen Akteuren festgelegt werden können. Technische Details können nicht Teil einer Verordnung sein, weil sie die dynamische Entwicklung des Patientendossiers als statische Elemente behindern werden. Unter diesen Voraussetzungen sieht die FMH das elektronische Patientendossier als Chance und freut sich auf die weitere Mitgestaltung. 